Elsevier required licence: (c) 2018. This manuscript version is made available under the CC-BY-NC-ND 4.0 license http://creativecommons.org/licenses/by-nc-nd/4.0/ 


\section{Influence of surface composition on the colloidal stability of ultra-small nanodiamonds in biological media}

Carlo Bradac, ${ }^{* * \dagger}$ Ishan Das Rastogi, ${ }^{b \dagger}$ Nicole M. Cordina,${ }^{c}$ Alfonso Garcia-Bennett ${ }^{b, c}$ and Louise J. Brown ${ }^{\text {** }}$

${ }^{\text {a }}$ School of Mathematical and Physical Sciences, University of Technology Sydney, Ultimo, NSW 2007, Australia.

${ }^{\mathrm{b}}$ Department of Chemistry and Biomolecular Sciences, Macquarie University, Sydney, NSW 2109, Australia.

c ARC Centre for Nanoscale Biophotonics, Macquarie University, Sydney, NSW 2109, Australia.

ABBREVIATIONS: ND - nanodiamond, BSA - bovine serum albumin, HSA - human serum albumin, DND - detonated nanodiamond, DI - deionized, FTIR - Fourier transform infrared spectroscopy, DLS - dynamic light scattering

\footnotetext{
*Corresponding authors. E-mail: carlo.bradac@uts.edu.au (Carlo Bradac), louise.brown@mq.edu.au (Louise Brown)

${ }^{+}$Equal Contribution
} 


\begin{abstract}
Fluorescent nanodiamonds (NDs) are strong contenders as bio-labels for life science imaging, diagnostics and therapeutics. Ultimately, for their use in biomedical applications, their size should ideally be less than $10 \mathrm{~nm}$. Yet, even more critical for their specificity and efficient uptake in cellular systems, is their resilience to aggregation, which is dictated by their colloidal stability in complex, physiological environments. To this end, we characterize ultrasmall detonation NDs $(\sim 5 \mathrm{~nm})$ by examining their surface chemical profiles and stability in solutions of varying ionic strength and $\mathrm{pH}$. Using dynamic light scattering measurements, we demonstrate that ultra-small ND particles with chemically homogeneous and negatively charged surface profiles are more stable than positive particles under a broad range of simulated biological environments. We show that the colloidal stability of both positive and negative ultra-small NDs is improved by functionalization with bovine serum albumin. Based on these analyses, we propose and describe strategies for enhancing the overall colloidal stability of ultra-small NDs and their resilience to aggregation. Our findings provide a practical framework towards the reduction in size of the bio-conjugates employed to probe complex biological systems, and the advancement of bio-imaging techniques with minimal perturbation of the molecular trafficking in cellular and organelle systems.
\end{abstract}




\section{Introduction}

Fluorescent nanodiamond (ND) particles are exceptional objects which are being increasingly adopted in several biomedical applications including drug delivery, imaging and diagnostics. ${ }^{1-3}$ In the field of biological imaging, their low cytotoxicity, high quantum yield, long fluorescence lifetime and stable photoluminescence set them apart from many other competitive systems currently employed as bio-labels. ${ }^{4-6}$ However, one notable challenge for using NDs as bio-labels is their strong and undesirable tendency to aggregate whilst in complex physiological environments.

When nanomaterials enter biological environments, the biomolecules - mostly proteins compete for binding to the surface of the nanoparticle, ${ }^{7-9}$ forming a protein corona layer around it. The proteins which are most abundant are the first to become adsorbed onto the surface. Over time, they are replaced by proteins which have a higher affinity to the nanoparticles surface. ${ }^{10}$ This is known as the Vroman effect. A recent study showed that, while the amount of proteins around the nanoparticle may change over time, the composition of the protein corona remains essentially unchanged. ${ }^{11}$ This indicates that the biomolecular layer around the nanoparticle is most likely stable in composition: it is instead the interaction at the nanoparticle bio-interface that dominates the binding dynamics in a complex and nontrivial way.

The biological behaviour of nanoparticles in solution is ultimately determined by their physico-chemical properties and - markedly - these tend to depend on many factors including surface chemistry profile of the nanoparticles, presence of salts, and $\mathrm{pH}$ of the surrounding solution. This is important as the behaviour and fate of nanoparticles is too often overly idealised in practical situations, such as cellular systems, where it can be difficult to anticipate the effect of complex environmental conditions on the colloidal behaviour of the nanoparticles. Strategies to improve the stability of nanoparticles do exist, yet they tend to compromise the functioning efficiency of the nanoparticle with respect to target molecules. ${ }^{12}$

Nanodiamond particles are no exceptions; methods to control their size and surface functionalisation are readily available, but effective approaches to manipulate their colloidal stability is lacking. This is especially true for ultra-small NDs, and detonation ND in particular where strongly bound agglomerates usually form if no countermeasures are taken. ${ }^{13}$ In general, the colloidal stability of NDs is electrostatically stabilized by increasing the surface charge density, which subsequently results in increased protein adsorption. ${ }^{14}$ The 
surface functionalisation of nanodiamonds by oxidation, ${ }^{15}$ or by binding blocking agents such as bovine serum albumin (BSA) ${ }^{16}$ and polyglycerol, ${ }^{17}$ have all been shown to change the charge density, as well as increase the chemical homogeneity of the NDs' surface. However, the majority of the biomolecule functionalisation studies reported in the literature typically have used ND material with particle sizes significantly larger than 10-20 nm. Lastly, oxidation, in particular, has been reported to also improve the hemocompatible properties of the ND particles ${ }^{18}$ - a prerequisite if they are to be used in biomedical contexts.

In this work, we present a systematic study of the role of the changing environment of surface functionalisation parameters on the overall colloidal stability of NDs in aqueous suspensions. We focus specifically on ultra-small NDs $(<10 \mathrm{~nm})$ which are most promising for bio-applications because of their small size. The high curvature of their surface decreases the protein-protein interactions among the biomolecules adsorbed on the nanoparticle's surface, resulting in fewer conformational changes in the attached proteins. ${ }^{16,19}$ Their high mobility and high surface-to-volume ratio (optimal for surface functionalisation), are also ideal for in vivo bio-imaging and drug delivery - provided that their colloidal stability and dispersibility are preserved once in biological systems. ${ }^{20,21}$ This is relevant, as the very same high surface-to-volume ratio makes NDs more susceptible to aggregation in the presence of salt $^{22}$ and other bio-components in the immediate environment.

Ultra-small NDs are usually produced by detonation, ${ }^{23}$ and typically form tight clusters which can only be de-aggregated through specific approaches including sonication, fractionation by centrifugation, ${ }^{24}$ plasma treatments, ${ }^{25,}{ }^{26}$ thermal oxidation ${ }^{27}$ or chemical processes. ${ }^{28}$ The colloidal stability for large clusters (size $\sim 30-60 \mathrm{~nm}$ ) of ultra-small detonation NDs with primary particle size of $\sim 5 \mathrm{~nm}$ has been previously studied over a range of $\mathrm{pH}$ values through their zeta potential variation. ${ }^{29}$ However, an in-depth analysis has never been conducted for individually-dispersed detonation NDs. Here we present a systematic analysis of the factors that lead to improved dispersibility and colloidal stability of monosized detonated nanodiamonds (DNDs), which are 5-6 nm in size, in complex physiological media. We use dynamic light scattering (DLS) measurements to determine the aggregation tendency of the isolated monodispersed DND particles of different surface chemical profiles in solutions over a broad range of $\mathrm{pH}$ values ( 2 to 12 ) and ionic strengths (up to $1 \mathrm{M} \mathrm{NaCl}$ ). Our findings suggest that the ND surface homogeneity plays a key role on the colloidal stability of the DND nanoparticle. Specifically, we find that when DNDs are used in complex media of high ionic strength such as phosphate buffer saline (PBS) - a commonly used isotonic buffered 
salt solution - the colloidal stability is significantly reduced. Finally, we propose and discuss strategies, such as surface functionalisation of the DNDs, to improve their overall stability and resilience to aggregation.

\section{Experimental}

\subsection{ND material and surface characterisation (FTIR \& XRD)}

Positively and negatively charged detonation nanodiamond (DND) colloidal suspensions (ND-H2O-5P, ND-H2O-5N, respectively), of average particle size of $\sim 5 \mathrm{~nm}$ in water, were obtained from Adámas Nanotechnologies (Raleigh, North Carolina, USA). The DND solutions $(10 \mathrm{mg} / \mathrm{mL})$ were bath-sonicated for 30 minutes prior to use.

To characterise the surface groups by attenuated total reflectance infrared spectroscopy (ATR-FTIR) using a Nicolet ${ }^{\mathrm{TM}}$ iS $10^{\mathrm{TM}}$ (Thermo Scientific), the positive and negative DND samples were prepared by drying $\sim 10 \mathrm{mg}$ of the ND powder in a hot-air oven at $100{ }^{\circ} \mathrm{C}$ for 30 minutes. FTIR spectra were collected under nitrogen to minimise the effect of atmospheric carbon dioxide. FTIR spectral analysis was performed within the wave number range 500$4000 \mathrm{~cm}^{-1}$.

X-ray diffraction (XRD) measurements were also conducted on the DND powders. The samples were deposited on a low-background silicon holder via evaporation, and analysed using a Bruker D8 Discover diffractometer using $\mathrm{Cu} \mathrm{K \alpha}$ radiation $(\lambda=1.5418 \AA)$ at $45 \mathrm{kV}$ and $35 \mathrm{~mA}$. The diamond (111) peak was used to analyse peak broadening in order to determine the crystallite size using Scherrer equation. Experimental XRD data were fitted using the software package High Score Plus (version 3 PANalytical). An internal standard was used to calibrate peak broadening.

\subsection{ND quantification of colloid (DLS \& zeta potential)}

A $2 \mathrm{mg} / \mathrm{mL}$ sample of the positive or negative DND particles were prepared in deionised (DI) water or potassium chloride $(\mathrm{KCl})$ solutions of increasing concentration $\left(1 \times 10^{-5}, 1 \times 10^{-4}\right.$, $1 \times 10^{-3}, 1 \times 10^{-2}, 1 \times 10^{-1}$ and $\left.1.0 \mathrm{M}\right)$. The DND suspensions were left at room temperature for $48 \mathrm{hrs}$ before measurement. All samples were then centrifuged by ultracentrifugation at 40,000 g for $8 \mathrm{hrs}$ in a fixed angle rotor (TLA 110 rotor, Optima MAX-XP Ultracentrifuge, Beckman Coulter) to remove large aggregates. Following ultracentrifugation, UV-Vis 
spectrophotometry was used to quantify the amount of monodispersed DNDs retained in solution relative to the amount of DNDs remaining in suspension for the DI-water-only sample. The absorbance of the solution at $400 \mathrm{~nm}$ was measured using a NanoDrop 100 spectrophotometer (Thermo Fisher Scientific). A relative yield was reported for samples under the conditions of varying salt concentrations.

The particle size distributions and zeta potentials for all DND colloids were performed by dynamic light scattering (DLS) using Zetasizer Nano ZS with a 633-nm laser source from Malvern Instruments. To account for the fact that in DLS measurements the signal from large aggregates tends to overshadow that of smaller particles (as the light scattered by a particle is proportional to the sixth power of its size) the data were transformed to number-weighted statistical distribution. The size distributions were measured from the average of 100 scans of $30 \mathrm{sec}$ each, and the zeta potentials were measured over 300 scans. Both size distribution and zeta potential were determined in the back-scattering configuration $\left(173^{\circ}\right)$.

Measurements of $\mathrm{pH}$ titration were conducted on an automated multipurpose titrator (MPT-2 accessory, Malvern instruments). The variation in size and zeta potential were recorded for both the positive and negative DND samples $(2 \mathrm{mg} / \mathrm{mL})$ from $\mathrm{pH} 2$ to 12 . For each of the samples, the starting $\mathrm{pH}$ titration point was the sample suspended in DI water.

\subsection{Functionalisation of DNDs with BSA}

The negatively and positively charged 5-nm DND samples were bath-sonicated for $30 \mathrm{~min}$ before the addition of bovine serum albumin (BSA). BSA $(5 \mathrm{mg} / \mathrm{mL})$ was prepared in $1 \mathrm{x}$ phosphate buffer saline (PBS, pH 7.2) and added to the DND ( $2 \mathrm{mg} / \mathrm{mL}$ ) to a final concentration of $2 \mathrm{mg} / \mathrm{mL}$. The samples were incubated for $90 \mathrm{~min}$ on a roller-mixer at $4{ }^{\circ} \mathrm{C}$. Following incubation, the samples were centrifuged at $10,000 \mathrm{~g}$ for $40 \mathrm{~min}$ and the BSA functionalised DNDs were collected in the pellet. FTIR spectra of the conjugated DNDs were also collected. The hydrodynamic diameter and zeta potential, for both the positive and negative conjugated DND particles in DI water and PBS, were recorded.

\section{Results}

3.1 Characterisation of ultra-small NDs - surface chemical functional groups 
Several synthetic approaches able to achieve ND particles with defined charge density and surface coverage are now commonly available. ${ }^{5}$ Diversity in the surface functionality of ND nanoparticles is desirable for target specificity. Yet, specific surface functionality also determines unique behaviour and distinct stability for the nanoparticles in solution, and this must be taken into account when engineering nanoparticle-bio-labeling complexes for any intended biological application.

Here we focus on ultra-small monosized detonated nanodiamonds (DNDs) of $\sim 5-6 \mathrm{~nm}$ in size. The X-ray diffraction (XRD) patterns confirmed the ultra-small nature of the DND samples used in this study (see Supplementary Figure S1). The XRD collected on the positive and negative DND samples showed a characteristic broad peak at $47.73^{\circ}$ in $2 \theta$, corresponding to the reflection from the (111) plane in the diamond lattice. No other diffraction peaks were observed. The crystallite size was estimated using the Scherrer equation to be 5.4 and $4.0 \mathrm{~nm}$ for positive and negative DNDs respectively, in agreement with the specifications from the manufacturer and the DLS data.

The FTIR spectra for the positive and negative DND samples used in this study are shown in Figure 1. For both samples, adsorbed water shows typical strong absorption bands in the range 3500-3300 $\mathrm{cm}^{-1}$ (vibrational mode) and at $1610 \mathrm{~cm}^{-1}$ (bending mode). ${ }^{29}$ Only the negative DND sample showed a prominent peak at $1780 \mathrm{~cm}^{-1}$. This peak corresponds to the carbonyl group $(\mathrm{C}=\mathrm{O})$ of carboxylic acid. The negative DNDs also show peaks corresponding to C-O-C vibrations and C-H bending, at $1450 \mathrm{~cm}^{-1}$ and $1080 \mathrm{~cm}^{-1}$, respectively. ${ }^{29-31}$ Compared to the negative DNDs, the relative lack of identifiable peaks in the FTIR profile of the positive DNDs sample suggests a higher density of graphitic impurities on the surface of the positive DNDs. ${ }^{27}$ Consistent with this is also the presence of pyrones $^{32}$ which likely result in the positive surface potential observed for these DNDs. ${ }^{4}$ Therefore, the positive DND sample has an overall inhomogeneous surface chemical profile with mostly phenols, pyrones and oxygen containing polyfunctional groups. In contrast, the negative sample is functionalised primarily with carboxylic groups with a more homogenous surface chemical profile. 


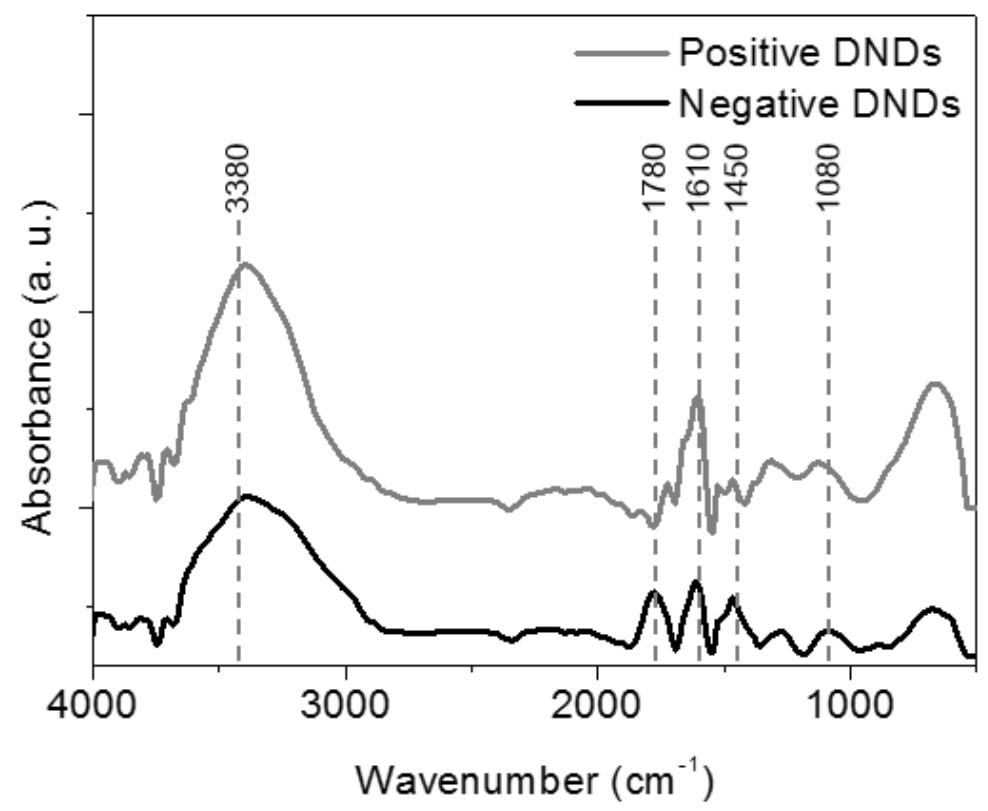

Figure 1. Surface chemical profiles of positive and negative DNDs. FTIR spectra of positively charged DNDs (grey) and negatively charged (black) DNDs ${ }^{33}$. Negative DNDs show a carbonyl peak at $\sim 1780 \mathrm{~cm}^{-1}$ representative of carboxyl groups. This peak is absent in positive DND's profile.

\subsection{Influence of surface chemical profile on ND aggregation in the presence of salt}

The colloidal stability of an aqueous resuspension of charged particles depends on the electrostatic repulsion between the particles. Characterization of the inherent surface charge of the nanoparticle, caused by the surface ions or functional groups, is commonly evaluated in terms of the particle's zeta potential. The zeta potential is a measure of the electric potential at the slipping plane in the double layer at the fluid-nanodiamond interface and measured relative to a point in the fluid away from the interface. Typically, for a suspension to remain in a suitably disperse state, a zeta potential value greater than $|30 \mathrm{mV}|$ is required. ${ }^{34}$ Both ion concentration and $\mathrm{pH}$ heavily factor into the stability of colloid suspensions.

In general, for both positive and negative charged nanoparticles, an increase in salt concentration (or ionic strength) in a solvent system results in reduced electrostatic repulsive forces causing the colloidal suspension to aggregate. ${ }^{35}$ The susceptibility of the positive and negative detonation NDs samples to salt-dependent aggregation for increasing ionic strength is shown in Figure 2. The [ grey]-coloured region indicates the zone where the nanodiamonds 
undergo notable aggregation (Supplementary, Figure S2), confirmed by zeta potential readings $(<|30 \mathrm{mV}|)$. As the ionic strength was increased, both the positive and negative DNDs showed an increase in their size [blue curve] with a simultaneous decrease in their zeta potential values [red curve].

The negative DNDs were observed to be more tolerant to increasing ionic strength than the positive DNDs. As confirmed by FTIR, overall, the negative DNDs displayed a more homogeneous surface chemical profile than the positive particles. Aggregation of the positive DNDs $\left(2 \mathrm{mg} / \mathrm{mL}\right.$ ) was observed at ionic strengths of $10^{-2} \mathrm{M}$ or higher (Figure $2 \mathrm{a}$ ) with visible phase separation of positive DNDs under gravity also observed at ionic strengths of $10^{-2} \mathrm{M}$ or higher (Supplementary, Figure S2a). In contrast, the negatively charged DNDs at the same, or higher ionic strengths, maintained better dispersibility properties (Figure 2b). Visible phase separation was only observed at concentrations of $10^{-1} \mathrm{M}$ or higher (Supplementary, Figure $\mathrm{S} 2 \mathrm{c})$.

Centrifugation was performed to provide a quantitative estimate of the colloidal stability of both the positive and negative suspension of DNDs under different ionic strengths, relative to water. Under the applied centrifugation forces, only monodisperse 5-nm DND particles would remain in suspension with large clusters and aggregated material sedimenting. In Figure 2c, the amount of DND content remaining in suspension post-centrifugation decreased under increasing ionic strength, as expected, in agreement with the DLS results. The negative DNDs [dark green curve] again showed better colloidal stability than positive DNDs [light green curve], as indicated by higher DND content in suspension at all ionic strengths. 

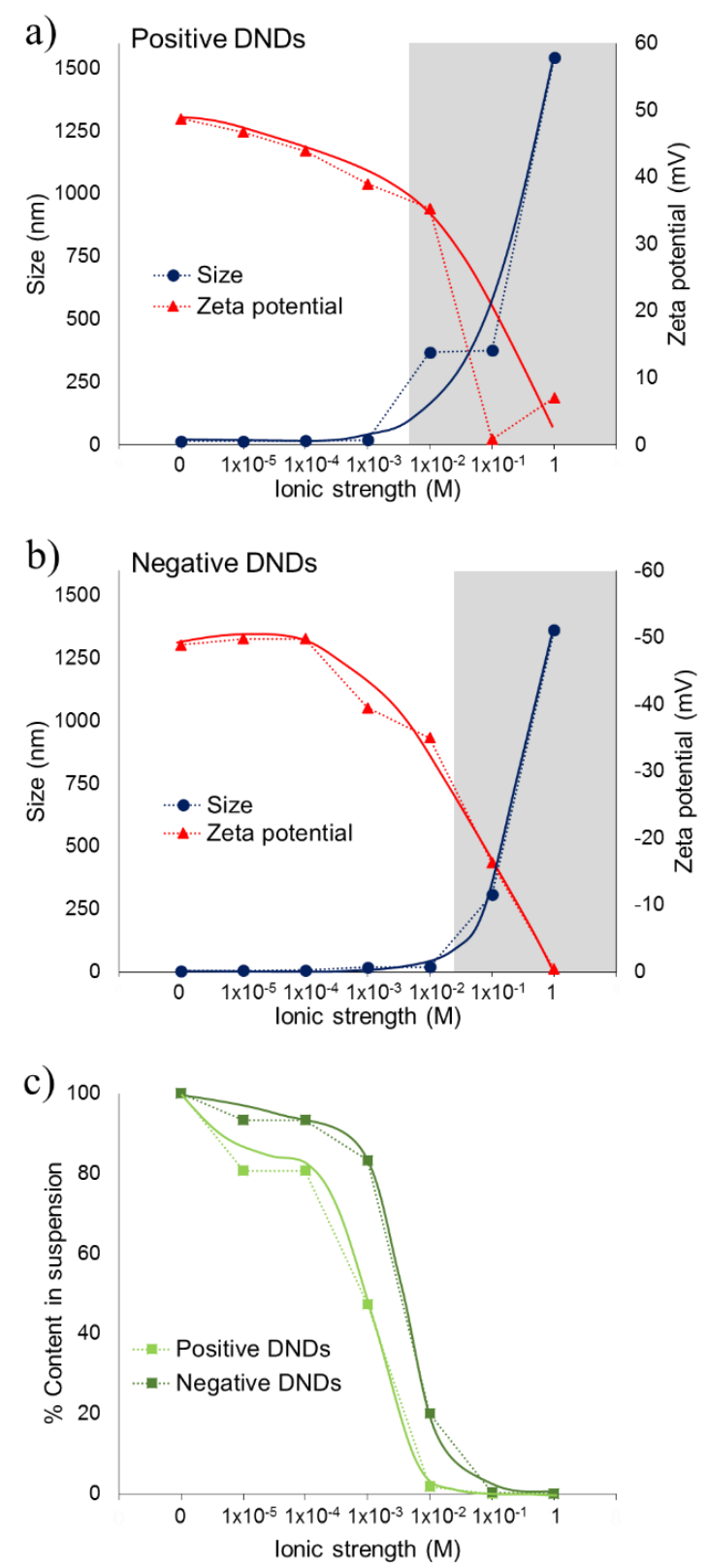

Figure 2. Size and zeta potential variation with increasing ionic strength for positive DNDs (a) and negative DNDs (b), and comparison of positive and negative DND content (\%) remaining in suspension with increasing ionic strength following ultra-centrifugation (c). DNDs of size $\sim 5 \mathrm{~nm}$ and zeta potential of $+45 \mathrm{mV}$ or $-45 \mathrm{mV}$ were suspended at $2 \mathrm{mg} / \mathrm{mL}$ in solvent of increasing ionic strength (up to $1 \mathrm{M}$ ). Following centrifugation at 40,000 $\mathrm{g}$ for $8 \mathrm{hr}$, the suspension was analysed by DLS to measure the average hydrodynamic diameter of the DND's in suspension (blue) and the zeta potential (red). Turbidity and aggregation of the DND suspension was observed for ionic strengths as indicated by the region shaded in grey. DND content was estimated from the absorbance at $400 \mathrm{~nm}$ using UV-Vis spectroscopy. 
The DLS sizing data showed that both the positive and negative DND samples maintained their ultra-small size range (average size of $\sim 5-6 \mathrm{~nm}$ ) up to ionic strengths of $10^{-4} \mathrm{M}$ (Figures $2 \mathrm{a}$ and $2 \mathrm{~b}$, blue curve). However, upon further increases in ionic strength beyond $10^{-4} \mathrm{M}$, the positive and negative DND suspensions were affected in different ways. For the positive DNDs, the colloidal suspension of particles at ionic strength of $10^{-3} \mathrm{M}$ visually appeared to be stable under gravity (Supplementary, Figure S2a). However, following ultra-centrifugation, greater than $50 \%$ of the population was pelleted (Figure 2c, light green curve). At this ionic strength, the size range of the particles remaining in solution, as detected by DLS, was $(19.1 \pm 4.8) \mathrm{nm}$. A modest decrease in the zeta potential from $(+48.7 \pm 11.7) \mathrm{mV}$ to $(+39.0 \pm 8.7) \mathrm{mV}$ was also noted (Figure $2 \mathrm{a}$, red curve). This slight increase in particle size is due to the formation of small aggregates or clusters of less than 5 monosized primary particles, each of 5-6 nm in dimension. Further increases in the ionic strength resulted in the rapid collapse of the stabilised population of clusters with larger particles of $(368 \pm 22) \mathrm{nm}$ in size forming at $10^{-2} \mathrm{M}$. These larger aggregates were subsequently sedimented and led to phase separation. Little DND content remained in suspension at these high ionic strengths $(\sim 10 \%)$ and further decreases in the zeta potential were noted.

For the negative DNDs in a solution of an ionic strength of $10^{-3} \mathrm{M}$ (Figure 2b), the size of the particles, $(18.2 \pm 4.5) \mathrm{nm}$, and the absolute value of the zeta potential, $(-39.5 \pm 7.3) \mathrm{mV}$, were similar to that observed for the positive DNDs at the same salt concentration. The small clusters of negative DNDs of $\sim 20 \mathrm{~nm}$ in size that formed at this same ionic strength were colloidally stable both under gravity and following centrifugation (Supplementary, Figure S2c and S2d, respectively), with $>95 \%$ of the DND content remaining in suspension (Figure $2 \mathrm{c}$, dark green curve). In contrast to the positive DNDs, at $10^{-2} \mathrm{M}$, the negative DNDs were still found in small cluster formations which were also stable under gravity (Supplementary Figure S2c). However, despite the resistance to aggregation, only $13 \%$ of the negative particles remained in suspension in this small cluster formation (Figure 2c). Beyond ionic strengths of $10^{-1} \mathrm{M}$, only large aggregates were found, with accompanying decreases in the magnitude of the zeta potential observed, as expected.

\section{$3.3 \mathrm{pH}$-dependent aggregation and its effect on colloidal stability of NDs}


The $\mathrm{pH}$ of the surrounding solution is a dominating environmental factor that influences the colloidal stability of a suspension (Figure 3). Like ionic strength, the colloidal stability at a given $\mathrm{pH}$ depends on the surface chemical profile of the DND particles. Specifically, the $\mathrm{pH}$ of the surrounding solution will influence the equilibrium between ND-O- $\mathrm{ND}-\mathrm{OH}$ and ND$\mathrm{OH}_{2}{ }^{+}$species. At low $\mathrm{pH}$, the $\mathrm{ND}-\mathrm{OH}_{2}{ }^{+}$species will be most dominant due to the adsorption of protons, whereas, the equilibrium will shift towards a higher population of $\mathrm{ND}^{-} \mathrm{O}^{-}$species at high $\mathrm{pH}$ due to the depletion of protons. The surface charge and hence the electrostatic stability will reach a minimum when the $\mathrm{pH}$ is close to the isoelectric point ( $\mathrm{pI}$ ) of the ND particle.

At low ionic strengths $\left(<10^{-3} \mathrm{M}\right)$, the isoelectric point for the suspension of positive DNDs was greater than $\sim 10$ (Figure 3c). For $\mathrm{pH}$ values below 10, the positive DNDs were either monodisperse or found in small stable clusters (Figure 3a) with zeta potential values $>30 \mathrm{mV}$ (Figure 3c). As the $\mathrm{pH}$ became more alkaline $(\mathrm{pH}>9)$, the positive DNDs were observed to undergo rapid aggregation with a corresponding decrease in the zeta potential to values $<30$ $\mathrm{mV}$. Under these alkaline conditions, the depletion of protons would contribute to the neutralisation of the charge of the positive DNDs. Further increases in $\mathrm{pH}$ values beyond $\mathrm{pH}$ 9 then led to total phase separation and to the corresponding further decreases in the zeta potential with readings reflecting a highly unstable suspension, $(+10.7 \pm 8.6) \mathrm{mV}$ at $\mathrm{pH} 12$. 

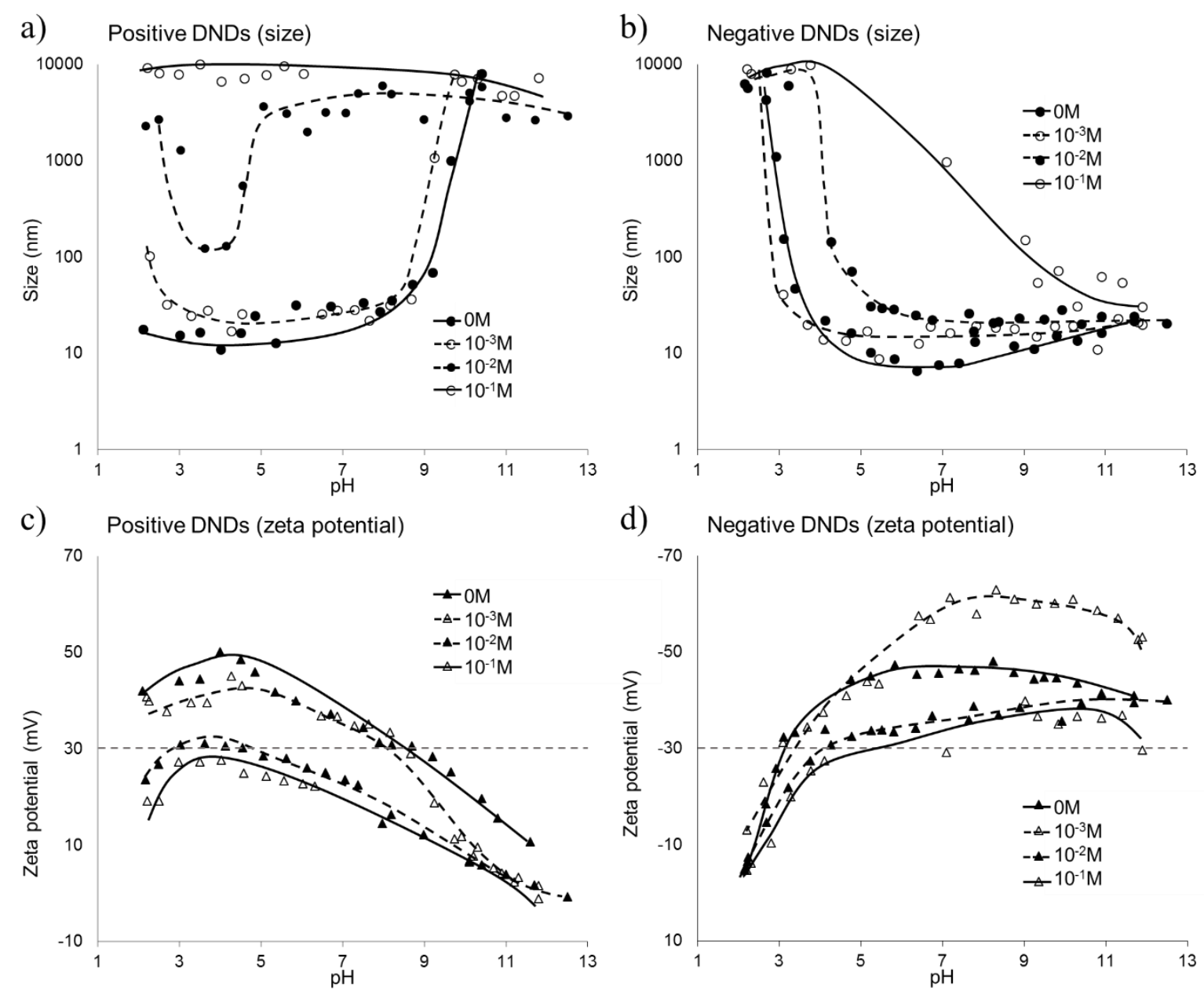

Figure 3. $\mathrm{pH}$ titration curves. $\mathrm{pH}$ profiles showing variation in size for (a) positive and (b) negative DNDs, and variation in zeta potential for (c) positive and (d) negative DNDs, in solutions of different ionic strengths $\left(0 \mathrm{M}, 1 \times 10^{-3} \mathrm{M}, 1 \times 10^{-2} \mathrm{M}, 1 \times 10^{-1} \mathrm{M}\right)$. The grey horizontal dashed line at $|30 \mathrm{mV}|$ represents the zeta potential threshold necessary to maintain colloidal stability.

In general, increasing the ionic strength of the solution decreased the $\mathrm{pI}$ of the positive DND suspension towards lower $\mathrm{pH}$ values (Figure 3c). At these progressively higher ionic strengths, the positive DNDs were more susceptible to $\mathrm{pH}$-dependent aggregation than the negative DNDs (Figure 3a). This can be seen by the narrower range of $\mathrm{pH}$ values for which the dimensions of the positive DNDs were reported to be small $(<20-30 \mathrm{~nm})$ and with stable zeta potential values of $>30 \mathrm{mV}$. Interestingly, a notable, but reproducible 'zone of stability' could be achieved for the positive DNDs in a suspension of high ionic strength $\left(10^{-2} \mathrm{M}\right)$ over the $\mathrm{pH}$ range of $3-5$. These well-defined conditions for the solvent could reversibly de- 
aggregate the large clusters formed at either side of this $\mathrm{pH}$ range. The small deflection of the zeta potential value, to a value just above the $30 \mathrm{mV}$ stability 'cut-off', reflected this finding.

For the negative DNDs, aggregation effects were observed when the $\mathrm{pH}$ of the resuspension became more acidic (Figure 3b). Like the positive DNDs, the negative DNDs showed higher susceptibility to $\mathrm{pH}$ dependent aggregation at progressively higher ionic strengths of the nano-environment. However, in contrast to the positive DNDs, the size analysis over the $\mathrm{pH}$ titration series showed that the negative DNDs maintained their monosized $(\sim 5 \mathrm{~nm})$ form over a wider range of $\mathrm{pH}$ values (Figure $3 \mathrm{~b}$ ). Although $\mathrm{pI}$ values cannot be accurately determined over the range of $\mathrm{pHs}$ used in these experiments $(2<\mathrm{pH}<12)$, we found that, upon increasing the ionic strength from $0 \mathrm{M}$ to $10^{-2} \mathrm{M}$, the range of $\mathrm{pH}$ for which the zeta potential was greater than $30 \mathrm{mV}$ went from $\mathrm{pH}=\sim 3-12$ for $0 \mathrm{M}$ to $\mathrm{pH}=\sim 5-12$ for $10^{-2} \mathrm{M}$. Naturally, more protons are necessary for charge compensation for these negative nanoparticles. Further, chloride ions may specifically adsorb onto the ND nanoparticles. This would further increase the NDs negative surface charge and so improve their colloidal stability at high ionic strengths (10 mM), even up to $100 \mathrm{mM}$. Similar observations have also been reported for gold nanoparticles with an overall negative surface charge. ${ }^{36}$

Taken together, this systematic study of $\mathrm{pH}$ and ionic strength demonstrates that the minimum absolute zeta potential value required to maintain colloidal stability of either the positive or negative DND suspensions is $\sim 30 \mathrm{mV}$. These findings can be used for optimising in-vitro reactions where the surface of the ND particles is to be modified, such as through EDAC/NHS coupling reactions. ${ }^{37}$ Tailored approaches should then be adopted when working with either positive or negative nanodiamonds as the starting material. However, the state of the stable colloid must also be considered when working with ultra-small DND particles. While we show that the stable colloid could represent a monodisperse suspension of primary particles in their ultra-small form (5-6 nm), the DNDs may also be found in stable clusters of as small as a few tens of $\mathrm{nm}$ up to $\sim 100 \mathrm{~nm}$ in size. Furthermore, in a solution of $10^{-1} \mathrm{M}$ ionic strength, both the positive and negative DNDs were found to not be stable at neutral $\mathrm{pH}$ but formed large unstable aggregates. As such, these particles are then not amenable for most biological applications in their raw form. Approaches to improve the stabilisation of DNDs that go beyond controlling the electrostatic interactions at the surface of the particle are therefore needed for these particles to be used in complex biological media, such as PBS. 


\subsection{Colloidal stability of BSA-functionalised DNDs in complex media}

The adsorption of macromolecules, such as synthetic polymers or biopolymers, onto the surface of nanoparticles can lead to a steric stabilisation of a colloid suspension. However, steric stabilisation is only likely to be effective if a uniform coating of the polymer is achieved on the surface of the particle. In order to improve the colloidal stability of DNDs in complex media such as PBS, the DNDs were functionalised with BSA. Figure 4 shows the FTIR profiles of the BSA functionalised positive (grey curve) and negative DNDs. ${ }^{33}$ The FTIR profiles suggested the successful functionalisation of both positive and negative DNDs with BSA (Figure 4). The profiles of both functionalised samples were very similar, with peaks at $\sim 3320 \mathrm{~cm}^{-1}$ indicating N-H vibrations. The strong peak at $\sim 1650 \mathrm{~cm}^{-1}$ corresponds to amide I band consisting of stretching vibrations of $\mathrm{C}=\mathrm{O}$ in the peptide bond; the peak at 1540 $\mathrm{cm}^{-1}$ indicates amide II band consisting of $\mathrm{N}-\mathrm{H}$ vibrations from the $\mathrm{C}-\mathrm{N}-\mathrm{H}$ groups of BSA. ${ }^{38}$

Both the BSA functionalised DNDs were suspended in $1 \times$ PBS (pH 7.2) and their colloidal stability compared to non-functionalised DNDs by recording the size and zeta potential for all samples (Table 1). The non-functionalised positive DNDs that were previously shown to be stable in water (Figure 2a) formed large aggregates in PBS with zeta potential values less than $|30 \mathrm{mV}|$. Aggregation was also observed for the BSA functionalised positive DNDs in water, as well as in PBS. This was not unexpected as the isoelectric point of BSA is $\sim 4.7 .^{39}$ At neutral $\mathrm{pH}$, the surface charge of the positive DNDs would be neutralised by the overall negative charge of the BSA, leading to aggregation of the complex both in water and in PBS.

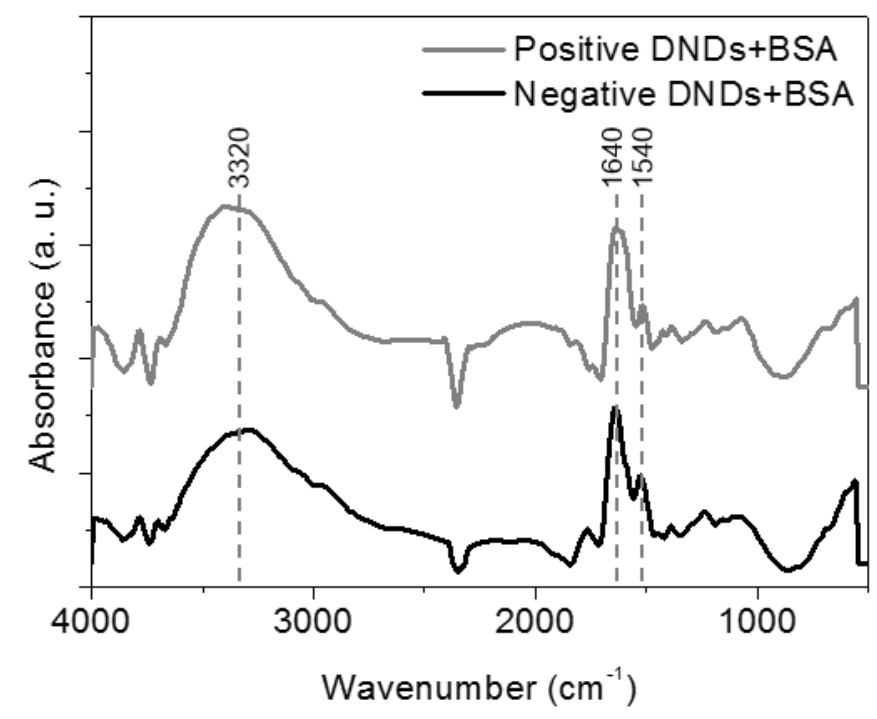


Figure 4. Surface chemical profiles of BSA-conjugated positive and negative DNDs. The FTIR spectra of BSA conjugated positively (grey) and negatively charged (black) DNDs ${ }^{33}$ show similar spectra with characteristic peaks for functional groups of BSA.

The non-functionalised negative DNDs, that were stable in water, aggregated in PBS. On the other hand, the BSA-functionalised negative DNDs formed a stable colloidal suspension in water as well as in PBS. The suspension of the negative DND-BSA complex in water was shown to have a hydrodynamic radius of $\sim 36 \mathrm{~nm}$, with a stable zeta potential value of $45.6 \mathrm{mV}$. While not as stable as in water, the same particles in PBS gave a slightly larger hydrodynamic radius of $\sim 68 \mathrm{~nm}$. This increase in diameter of the NDs, in the presence of BSA, indicated that the additional stability was due to the extra electrostatic repulsion provided as a result of the formation of a surface layer of adsorbed BSA protein molecules around the nanoparticle. Although still only an ideal case, the uniformly coated surface of BSA molecules, which provided a different partial surface charge to the NDs, was sufficient to overcome the progressive destabilisation of particles as the ionic strength of the surrounding solution increased. While colloid stability of aqueous suspensions are often explained by the classic Derjaguin-Landau-Verwey-Overbeek (DLVO) theory, ${ }^{40}$ our differing results demonstrate that NDs can indeed be restabilised at high ionic strengths by 'capping' with an appropriately charged protein biomolecule.

\begin{tabular}{|c|c|l|l|l|l|}
\hline \multicolumn{2}{|c|}{} & \multicolumn{1}{|c|}{ Size (nm) } & $\begin{array}{c}\text { Zeta potential } \\
(\mathbf{m V})\end{array}$ & PDI \\
\hline \multirow{2}{*}{$\begin{array}{c}\text { Positive } \\
\text { DNDs } \\
\text { conjugated }\end{array}$} & \multirow{2}{*}{\begin{tabular}{c} 
BSA \\
\cline { 3 - 6 }
\end{tabular}} & PBS & $4201 \pm 459$ & $+5.3^{\dagger}$ & 1.00 \\
\cline { 2 - 6 } & \begin{tabular}{c} 
conjugated \\
\cline { 3 - 6 }
\end{tabular} & PBS & $2317 \pm 240$ & $-10.3^{\dagger}$ & $0.18^{*}$ \\
\hline Negative & non & water & $4.7 \pm 1.4$ & $-48.9 \pm 8.1$ & 0.71 \\
\hline
\end{tabular}




\begin{tabular}{|c|l|l|l|l|l|}
\hline DNDs & conjugated & PBS & $2107 \pm 265$ & $-21.1^{\dagger}$ & 0.80 \\
\cline { 2 - 6 } & \multirow{2}{*}{$\begin{array}{c}\text { BSA } \\
\text { conjugated }\end{array}$} & water & $35.8 \pm 6.4$ & $-45.6 \pm 7.6$ & $0.27^{*}$ \\
\cline { 3 - 6 } & & PBS & $67.8 \pm 11.0$ & $-16.7^{\dagger}$ & $0.37^{*}$ \\
\hline
\end{tabular}

Table 1. Effect of BSA conjugation on colloidal stability of positive and negative DNDs in water and $1 \times$ PBS (high ionic strength). Polydispersity index values (PDI) range from 0 to 1 and reflect how 'monodispersed' a suspension is, with lower values $(<0.3)$ indicating a more monodispersed distribution of particles. The ‘*, represents a stable colloid, while “†' indicates zeta potential values where the standard deviation could not be reliably determined due to the high ionic strength of the sample.

\subsection{Improved colloidal stability due to homogenous surface chemical profile}

Ultra-small DNDs have been shown to have inherent surface inhomogeneity features arising from the presence of low and high index facets on their surface. ${ }^{4,26}$ The surface energies of these facets vary, and therefore their termination is energetically favoured by several and different surface groups. ${ }^{41}$ This leads to inhomogeneous surface chemical profiles of DNDs, as we observed for the positive DNDs. Naturally, the functionalisation with one type of chemical group onto the DND surface can improve surface homogeneity as observed for negative DNDs. In turn, particles with a homogenous surface are more resistant to aggregation solutions of increasing ionic strengths and varying $\mathrm{pH}$. Our results demonstrated this link between surface homogeneity and colloid stability of DNDs with superior stability observed for the negative DNDs compared to the positive. However, achieving a perfect uniform coverage of the surface by the same or similar charged chemical groups is energetically difficult to achieve due to morphological distortions on the surface of these

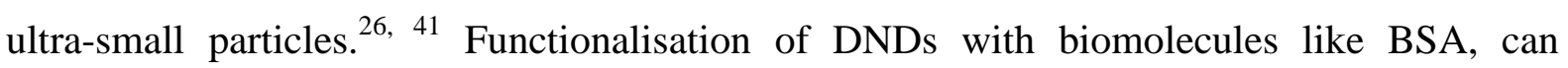
therefore be used to mask such surface inhomogeneities and also provide for a uniform polymer-like coating of a uniform charge, thereby improving the stability of ultra-small DNDs in complex solutions, such as PBS. While our BSA-conjugated particles are stable for up to 1 week at $4{ }^{\circ} \mathrm{C}$, long-term stability can also be achieved through cost-effective strategies including freeze-drying in the presence of cryoprotectants such as sugars. ${ }^{42}$ 


\section{Conclusions}

Ideal bio-label candidates for bio-medical imaging and therapeutics are required to maintain their dimensions and functional identity on a molecular level. Knowledge of the stability i.e. the propensity to aggregate - of ultra-small NDs in complex physiological fluids is paramount. Yet, predicting the NDs' stability in varying solutions is difficult and requires a complete description of the particles' surface properties. The available theoretical models are indeed powerful tools to describe the complex ND/environment interplay; they account for factors such as zeta potential, ionic strength and $\mathrm{pH}$ of the solvent systems along with surface composition, but they often tend to over-idealise their overall behaviour. More practical and systematic approaches - like those presented here - are thus a powerful addition when assessing the colloidal stability of nanoparticles in different environments.

Specifically, while the size of nanoparticles typically influences their stability - with smaller particles being more prone to aggregation than larger ones - our results demonstrate that positive NDs have a greater propensity to aggregate in solution than negative ND particles of the same size. Particles with a more homogeneous surface chemical profile, as in the case of the negative DNDs, were shown to be more resistant to forming aggregates and exhibited a superior colloidal stability over a broader range of salt concentrations and $\mathrm{pH}$ values. The role of surface homogeneity becomes more critical for resuspensions in high ionic strengths such as that present in biological media like PBS. Further functionalisation with biomolecules like BSA can be done to mask surface variations, thereby improving colloidal stability in complex environment of PBS. Our study is a step towards engineering better nanodiamondbased bio-labels, where the choice of an optimal chemical profile allows for the NDs to be as small as $5 \mathrm{~nm}$, yet without compromising their colloidal and functional stability even in complex physiological environments. 


\section{Acknowledgements}

The authors acknowledge support from the Nanoscale Biophotonics (CE140100003), and a Macquarie University Research Development Grant to L.J.B. A.G.B is currently an ARC Future Fellowship (FT150100342). C.B. is on a UTS Chancellor's Postdoctoral Research Fellowship. 


\section{References}

1. D. Ho, ACS Nano, 2009, 3, 3825-3829.

2. E. Perevedentseva, Y. C. Lin, M. Jani and C. L. Cheng, Nanomedicine (Lond), 2013, 8, 2041-2060.

3. V. Vaijayanthimala, D. K. Lee, S. V. Kim, A. Yen, N. Tsai, D. Ho, H.-C. Chang and O. Shenderova, Expert. Opin. Drug Deliv., 2015, 12, 735-749.

4. A. M. Schrand, S. A. C. Hens and O. A. Shenderova, Crit. Rev. Solid State, 2009, 34, 18-74.

5. J. M. Say, C. Vreden, D. J. Reilly, L. J. Brown, J. R. Rabeau and N. J. C. King, Biophys. Rev., 2011, 3, 171-184.

6. V. N. Mochalin, O. Shenderova, D. Ho and Y. Gogotsi, Nat. Nano., 2012, 7, 11-23.

7. M. P. Monopoli, F. B. Bombelli and K. A. Dawson, Nat. Nano., 2011, 6, 11-12.

8. M. P. Monopoli, D. Walczyk, A. Campbell, G. Elia, I. Lynch, F. Baldelli Bombelli and K. A. Dawson, J. Am. Chem. Soc., 2011, 133, 2525-2534.

9. C. D. Walkey and W. C. W. Chan, Chem. Soc. Rev., 2012, 41, 2780-2799.

10. L. Vroman, Adams, A. L., Fischer, G. C., and Munoz, P. C., Blood, 1980, 55, 156159.

11. S. Tenzer, D. Docter, J. Kuharev, A. Musyanovych, V. Fetz, R. Hecht, F. Schlenk, D. Fischer, K. Kiouptsi and C. Reinhardt, Nat. Nano., 2013, 8, 772-781.

12. R. A. Sperling and W. Parak, Philosophical Transactions of the Royal Society of London A: Mathematical, Physical and Engineering Sciences, 2010, 368, 1333-1383.

13. A. Krüger, F. Kataoka, M. Ozawa, T. Fujino, Y. Suzuki, A. E. Aleksenskii, A. Y. Vul and E. Osawa, Carbon, 2005, 43, 1722-1730.

14. A. Gessner, A. Lieske, B. R. Paulke and R. H. Müller, Eur. J. Pharm. Biopharm., 2002, 54, 165-170.

15. A. Krueger and D. Lang, Adv. Funct. Mater., 2012, 22, 890-906.

16. S. L. Masoud Rahman, Nancy Tawil, L'Hocine Yahia, Morteza Mahmoudi, in Protein-Nanoparticle Interactions The Bio-Nano Interface, Springer Series in Biophysics, 2013, vol. 15.

17. L. Zhao, A. Shiino, H. Qin, T. Kimura and N. Komatsu, J. Nanosci. Nanotechnol., 2015, 15, 1076-1082.

18. L. Kuang-Kai, C. Chia-Liang, C. Chia-Ching and I. C. Jui, Nanotechnology, 2007, 18, 325102.

19. I. Lynch, A. Salvati and K. A. Dawson, Nat. Nano., 2009, 4, 546-547.

20. Y. Yuan, Y. Chen, J.-H. Liu, H. Wang and Y. Liu, Diam. Relat. Mater., 2009, 18, 95100.

21. K. Purtov, A. Petunin, E. Inzhevatkin, A. Burov, N. Ronzhin, A. Puzyr and V. Bondar, J. Nanosci. Nanotechnol., 2015, 15, 1070-1075.

22. T. Yoshikawa, V. Zuerbig, F. Gao, R. Hoffmann, C. E. Nebel, O. Ambacher and V. Lebedev, Langmuir, 2015, 31, 5319-5325.

23. V. Y. Dolmatov, M. Veretennikova, V. Marchukov and V. Sushchev, Phys. Solid State, 2004, 46, 611-615.

24. Y. Morita, T. Takimoto, H. Yamanaka, K. Kumekawa, S. Morino, S. Aonuma, T. Kimura and N. Komatsu, Small, 2008, 4, 2154-2157.

25. I. Petrov, O. Shenderova, V. Grishko, V. Grichko, T. Tyler, G. Cunningham and G. McGuire, Diam. Relat. Mater., 2007, 16, 2098-2103.

26. O. A. Shenderova and G. E. McGuire, Biointerphases, 2015, 10, 030802.

27. S. Osswald, G. Yushin, V. Mochalin, S. O. Kucheyev and Y. Gogotsi, J. Am. Chem. Soc., 2006, 128, 11635-11642. 
28. C. Bradac, T. Gaebel, N. Naidoo, M. J. Sellars, J. Twamley, L. J. Brown, A. S. Barnard, T. Plakhotnik, A. V. Zvyagin and J. R. Rabeau, Nat. Nano., 2010, 5, 345349.

29. N. Gibson, O. Shenderova, T. J. M. Luo, S. Moseenkov, V. Bondar, A. Puzyr, K. Purtov, Z. Fitzgerald and D. W. Brenner, Diam. Relat. Mater., 2009, 18, 620-626.

30. X. Xu, Z. Yu, Y. Zhu and B. Wang, J. Solid State Chem., 2005, 178, 688-693.

31. X. Xu, Z. Yu, Y. Zhu and B. Wang, Diam. Relat. Mater., 2005, 14, 206-212.

32. E. Ōsawa, Diam. Relat. Mater., 2007, 16, 2018-2022.

33. P. Wilson, S. Blackburn, R. Greenwood, B. Prajapti and K. Smalley, J. Eur. Ceram. Soc., 2011, 31, 1849-1855.

34. J. D. Clogston and A. K. Patri, in T Characterization of Nanoparticles Intended for Drug Delivery, 2009, vol. 697, pp. 63-70.

35. N. I. Lebovka, in Polyelectrolyte Complexes in the Dispersed and Solid State I: Principles and Theory, ed. M. Müller, Springer Berlin Heidelberg, Berlin, Heidelberg, 2014, DOI: 10.1007/12_2012_171, pp. 57-96.

36. C. Pfeiffer, C. Rehbock, D. Huhn, C. Carrillo-Carrion, D. J. de Aberasturi, V. Merk, S. Barcikowski and W. J. Parak, J. R. Soc. Interface, 2014, 11, 20130931.

37. C. Bradac, J. M. Say, I. D. Rastogi, N. M. Cordina, T. Volz and L. J. Brown, J. Biophotonics, 2016, 9, 296-304.

38. J. Grdadolnik and Y. Maréchal, Biopolymers, 2001, 62, 40-53.

39. S. Ge, K. Kojio, A. Takahara and T. Kajiyama, J. Biomater. Sci. Polym. Ed., 1998, 9, 131-150.

40. B. Derjaguin, Kolloid-Zeitschrift, 1934, 69, 155-164.

41. K. Larsson, in Novel Aspects of Diamond: From Growth to Applications, ed. N. Yang, Springer International Publishing, Cham, 2015, DOI: 10.1007/978-3-319-09834-0_3, pp. 53-83.

42. W. Abdelwahed, G. Degobert, S. Stainmesse and H. Fessi, Advanced Drug Delivery Reviews, 2006, 58, 1688-1713. 